historical property that makes certain objects special. Critically, children as young as age 4 reason about authentic objects in an essentialist manner (Frazier \& Gelman 2009; Gelman et al. 2014). Because the essencelike effects of ownership appear early and persist into adulthood, it is unlikely that they are some later by-product of psychological essentialism. Thus, at least some element of historical information must be accounted for by any comprehensive explanation of psychological essentialism.

Historical information plays an important role in considerations of ownership and authenticity, a role that highlights several gray areas with respect to the inherence heuristic. First, is the separation between intrinsic and extrinsic information detailed by $C \& S$ meaningful? There are at least two effects of this dichotomy on the model: (1) a separation between internal features (e.g., DNA, insides, essences) and external features (e.g., conventions, surface details), and (2) a division between features that are salient and accessible and those that are not. These two elements travel together in the examples presented by $C \& S$, but ownership is a case where the two might be split apart, unless one conceptualizes ownership in a very specific way (i.e., as an intrinsic feature that is historically, and therefore externally, defined - but whether this is possible is an empirical question).

Second, can historical information play a role in the output of the inherence heuristic? Historical information plays a critical role in defining property (Gelman et al. 2012) and authentic objects (Frazier \& Gelman 2009). Ownership information is accessible and salient in exactly the way that other kinds of historical information, such as social conventions and historical events, are not. In its current formulation, the inherence heuristic states that the mental shotgun selects inherent features because they are salient and accessible, but there is no requirement that they be internal or intrinsic. Although it may be true that inherent features are overrepresented in human explanation, perhaps because they are salient and accessible, does the model need to be limited to inherent features?

Finally, can the inherence heuristic effectively serve as the foundation for psychological essentialism if it accounts only for essentialist thinking within a subset of domains? The inherence heuristic is a process that is proposed to explain broad patterns, and the relationship between individuals and property is consistent and systemic. More specifically, people treat property as though it is imbued with the essence of current and prior owners. Historical information is central to concepts of ownership, and historical path may be an important element of essentialism, especially with respect to artifacts (see Frazier \& Gelman 2009). However, in its current formulation, the inherence heuristic does not account for authenticity and other effects of ownership. Thus, the inherence heuristic appears to be well situated to address how we think about the intrinsic properties that underlie many human intuitions, but if this process is truly the foundation for psychological essentialism, then it needs to address historical information in some contexts.

\section{The social aetiology of essentialist beliefs}

\author{
doi:10.1017/S0140525X1300383X
}

\section{Cliodhna O'Connor and Helene Joffe}

Division of Psychology \& Language Sciences, University College London, London WC1H OAP, United Kingdom.

\section{oconnor.c@ucl.ac.uk h.joffe@ucl.ac.uk}

https://iris.ucl.ac.uk/iris/browse/profile?upi=COCON80

http://www.ucl.ac.uk/psychlangsci/research/CPB/people/cpb-staff/

h_joffe

\footnotetext{
Abstract: This commentary highlights the importance of attending to the sociocultural contexts that foster essentialist ideas. It contends that Cimpian \& Salomon's (C\&S's) model undervalues the extent to which the development of essentialist beliefs is contingent on social experience. The result is a restriction of the model's applicability to realworld instances of essentialism-fuelled prejudice and discrimination.
}

Psychological essentialism is a pervasive pattern of thinking that has been implicated in a host of unpleasant societal processes. As such, the endeavour of Cimpian \& Salomon $(C \& S)$ to elucidate the roots of this psychological tendency is an important undertaking. However, we argue that the model they have developed sustains one clear void: Namely, it underplays the influence of social factors on the development of essentialist beliefs, particularly when those beliefs pertain to social groups.

$\mathrm{C} \& \mathrm{~S}$ contend that the origins of psychological essentialism lie in the "inherence heuristic," which they present as a product of basic, potentially innate properties of the human cognitive system. Within their model of the development of essentialism, social factors are relegated to a subsidiary role. The basic tendency to make essentialist assumptions is cognitively prescribed, and social experience simply furnishes the surface content that allows these essentialist principles to be developed into culturally sensible narratives. However, this jars with the extensive body of theoretical and empirical literature that suggests that the process, as well as the content, of thought is socio-culturally shaped (Fiske et al. 1998). As children mature, they are socialised into a community of minds from whom they learn the appropriate ways of representing the world around them. This premise opens the possibility that patterns of essentialist thinking may be acquired, or at least formatively moulded, as a result of people's encounters with the social world. Indeed, research shows that the propensity to engage in essentialist thought (as well as the precise content of essentialist ideas) varies systematically across cultural groups, including those differentiated by religion (Birnbaum et al. 2010; Diesendruck \& Haber 2009), nationality (Mahalingam \& Rodriguez 2003), social status (Mahalingam \& Rodriguez 2006), political conservatism (Rhodes \& Gelman 2009), and race (Jayaratne et al. 2009). This suggests that social experience can affect the very impulse to think in essentialist terms, rather than merely supplying the narratives that are tacked onto the fundamental, cognitively given precepts.

C\&S acknowledge the proposition that essentialist thinking is a product of socialisation, but dismiss this hypothesis as implausible. The sole empirical grounds provided for this position relates to the failure of research to detect sufficient evidence of overt transmission of essentialism within parent-child conversations. Verbalised parent-child interaction is, however, an extremely narrow proxy for the whole spectrum of social influences on individual cognition. Engagement with the social world occurs through many channels - through institutions such as the mass media, education, commercial markets, and politics, as well as immediate verbal and non-verbal interpersonal interactions. All such means of communication are viable vectors of essentialist ideas.

As an example of one such channel of social influence, research has suggested that the popular diffusion of scientific knowledge can propagate essentialist representations of particular social groups (Dar-Nimrod \& Heine 2011). Recent research on media coverage of neuroscience shows that a key way in which neuroscience is communicated to the public is via emphasising that differences between social groups are biologically rooted (O'Connor et al. 2012). This neuro-essentialism of social categories is exemplified by the ubiquitous trope of "the [adjective] brain" (e.g., "the male brain," "the gay brain," "the criminal brain"), which expressly condenses the essence of a given category into the notion of a distinctive brain type that is universally shared by all category members. The proliferation of such messages within the public sphere may trigger essentialist thinking in those who encounter them. Experimental research has shown that exposing people to information about the purported biogenetic foundations of, for example, gender (Brescoll \& LaFrance 2004; Coleman \& Hong 2008) or race (Keller 2005; No et al. 2008; Williams \& Eberhardt 2008) exacerbates essentialist beliefs and corresponding processes of stereotyping and discrimination. Though C\&S acknowledge that biological information can be recruited into the "stories" that elaborate inherencebased assumptions, they do not consider whether exposure to 
Commentary/Cimpian \& Salomon: The inherence heuristic

this biological information might itself cultivate essentialist beliefs, which would not otherwise have emerged.

The model's relative neglect of social influence restricts its applicability to real-world instances of essentialism-fuelled prejudice and discrimination. Although the authors acknowledge that the content of essentialist beliefs can deviate between category domains, they do not engage with the evidence that different human categories attract varying levels of essentialism (Haslam et al. 2000; Joffe \& Staerklé 2007). An account of essentialism that hinges on a basic cognitive bias cannot elucidate why, for example, categories relating to eye colour do not elicit the level of essentialism that is directed at categories relating to gender or race. Without due attention to the modulating influence of societal processes, the model cannot address the key question of why particular social groups, within particular historical contexts, become constituted as homogeneous, immutable, and denigrated entities. Neither does it aid in discriminating between those contexts in which essentialist identities are internalised by marginalised groups, and cases where oppressive essentialist identities are actively challenged and resisted (Mahalingam 2007). Indeed, characterising essentialism as a product of instinctive cognitive mechanisms may lend an air of inevitability, obscuring the processes by which change can be effected in the essentialism that particular groups in society face.

Undoubtedly, essentialist beliefs are premised upon cognitive (and affective) patterns, and these merit empirical elaboration. However, social contexts shape how these basic cognitive tendencies manifest and hence their implications for individuals and society. In building an account of essentialism that foregrounds instinctual cognition to the exclusion of formative social factors, C\&S's model may itself exemplify an inherence-based explanation. It thereby risks eliding the social interests and agendas that selectively channel the malignancies of essentialism towards particular sectors of society.

\section{Is psychological essentialism an inherent feature of human cognition?}

doi:10.1017/S0140525X13003841

\section{Christopher Y. Olivola ${ }^{a}$ and Edouard Machery ${ }^{b}$ \\ a'Tepper School of Business, Carnegie Mellon University, Pittsburgh, PA 15213; ' ${ }^{\mathrm{b}}$ Department of History and Philosophy of Science, University of Pittsburgh, Pittsburgh, PA 15260. \\ colivola@andrew.cmu.edu machery@pitt.edu \\ https://sites.google.com/site/chrisolivola/ \\ http://www.pitt.edu/ machery/}

\begin{abstract}
Recent evidence shows that psychological essentialism is neither a universal nor stable feature of human cognition. The extent to which people report essentialist intuitions varies enormously across cultures and education levels, and is also influenced by subtle, normatively irrelevant contextual manipulations. These results challenge the notion that the human mind is "fitted" with a built-in inherence heuristic that produces essentialist intuitions.
\end{abstract}

A major goal of the article by Cimpian \& Salomon (C\&S), as stated by the authors themselves (see sect. 4), is to argue that their proposed inherence heuristic provides the cognitive foundation on which psychological essentialism emerges. In doing so, the authors seem to take it as a given that psychological essentialism is a fundamental feature of the human mind that needs to be explained.

However, recent evidence suggests that psychological essentialism may not be a universal or immutable human tendency. In a recent set of studies (Machery et al., submitted), we examined the stability of psychological essentialism. The participants in our studies were asked, for example, whether lemons that undergo a chemical transformation, which alters their exterior properties (e.g., taste and appearance) but conserves their genetic structure, would still be considered lemons, or (conversely) whether a liquid discovered on a distant planet that shares all the functional and superficial properties of water, despite having a completely different molecular structure, should be called "water." An essentialist position would define a category of things (or kind) by their stable, underlying properties. Thus, according to a true essentialist, lemons that undergo a superficial transformation while retaining their genetic structure would still be lemons, whereas the alien liquid would not be considered water (since its molecular structure is different) regardless of how closely it resembles, tastes like, or fulfills the same role as water. Thus, by tallying the proportion of participants who believe the lemon remains a lemon in the chemical transformation scenario, or that the alien liquid is not water in the discovery scenario, we can estimate which proportion of a given sample holds essentialist beliefs.

The first study considered essentialist intuitions in 10 different nations spread across four continents. We found that the likelihood of holding essentialist intuitions varied dramatically (and significantly) across cultures, from less than $10 \%$ of participants in a particular country holding essentialist beliefs to more than $70 \%$ of those in another country holding these intuitions. Furthermore, these intuitions were found to vary significantly with gender and education. A second study showed that the type of scenario (transformation vs. discovery) and the category (organic/biological vs. physical/chemical) both significantly (and independently) influenced the likelihood that participants reported essentialist intuitions. Finally, a third study showed that merely varying how we framed the (same) question in a transformation scenario significantly altered the likelihood that participants would report essentialist intuitions.

Taken together, the aforementioned studies show that, far from being universal, essentialist beliefs vary dramatically across cultures, gender, and educational levels - much like many other philosophical intuitions (e.g., Machery et al. 2004; 2009). These studies also show that, far from being stable, the likelihood that people hold essentialist intuitions varies considerably with normatively irrelevant contextual factors, such as the type of scenario used to elicit beliefs or the way the question is being framed.

The results of our studies are consistent with a small, but important, literature on cross-cultural variation in psychological essentialism and on the role of social factors in the prevalence of essentialism. There is substantial variation regarding which properties are thought to be immutable as well as caused by internal features. Pfeffer et al. (1998) found that British children are more likely than Nigerian children to view criminal behavior as immutable and internally caused. Giles et al. (2008) also found that Black South African children are more likely than African-American children to view aggression as inborn and immutable. Giles et al. argue that this finding is consistent with the social role of essentialism in justifying inequalities during apartheid (see also Mahalingam 2003b). There is also substantial variation across cultures and social groups regarding which categories are essentialized. Mahalingam (2003a) has shown that the Aravanis of India (a minority of biological men who dress and live as women) essentialize the female gender, but not the male gender, holding that a male can become a woman by cross-dressing. Mahalingam (2003b) has also provided evidence suggesting that Brahmins and Dalits in India essentialize the Indian castes differently. It would thus seem that, far from being a default mode of thinking, essentialism is used strategically, in a contextual manner, often to fulfill some political goals (see also Pereira et al. 2010).

In sum, a growing of body research suggests that psychological essentialism is neither a universal nor stable feature of human cognition. Consequently, this research challenges the notion that the human mind is "fitted" with a built-in inherence heuristic that produces essentialist intuitions.

\section{ACKNOWLEDGMENT}

Christopher Y. Olivola acknowledges the support of the British Academy and the Royal Society. 